\title{
Abnormal pitch-time interference in congenital amusia: Evidence from an implicit test
}

\author{
Micha Pfeuty \\ Université Victor Segalen, Bordeaux, France \\ AND \\ Isabelle Peretz \\ University of Montreal, Montreal, Quebec, Canada
}

\begin{abstract}
Congenital amusia, characterized by a severe problem in detecting anomalies in melodies, is a lifelong disorder that has been ascribed to an acoustical pitch deficit. In the present study, we investigated how the perception of a duration is altered when it is bounded by tones varying in pitch. The results show that temporal accuracy is impaired by pitch variations as small as a quarter of a semitone in control participants, whereas it is impaired only when pitch variations are increased to 4 semitones in congenital amusics. Furthermore, control participants associate intervals bounded by low- and high-pitched tones with long and short durations, respectively. Amusic participants do not make this connection, even with large pitch differences, pointing to a deficit in pitch-time integration. Thus, our results are consistent with the notion that congenital amusia is linked to a neurogenetic anomaly that impairs pitch processing, independently of task factors.
\end{abstract}

Humans are born with the potential both to speak and to make music. For the majority of those who are musically untrained, this fundamental human trait is expressed by avid listening and by occasional dancing and singing. The propensity to engage in music ultimately gives rise to a sophisticated music processing system that is largely acquired implicitly by experience (Peretz, 2006). However, a minority of individuals never acquire this core musical system, either in part or in total. This condition, termed congenital amusia (Peretz, 2001), affects $4 \%$ of the general population (Kalmus \& Fry, 1980) and is akin to other congenital developmental disorders, such as prosopagnosia, dyscalculia, dysphasia, and dyslexia, and is thought to result from a musical pitch disorder (Foxton, Dean, Gee, Peretz, \& Griffiths, 2004; Hyde \& Peretz, 2004).

The nature of the pitch disorder remains, however, unsettled. It has been shown that amusic individuals cannot detect pitch changes that are smaller than 1 semitone, which represents the difference in frequency between adjacent pitches in Western music and is the building block of musical scales. Yet, there are indications that amusic individuals might be able to use pitch information, albeit implicitly. Amusics are able to reproduce pitch directions vocally, although being unable to report these explicitly (Loui, Guenther, Mathys, \& Schlaug, 2008). Similarly, the brain of amusics responds to quarter-tone (i.e., 50-cent) pitch violations, by exhibiting an early right-lateralized negative electrical response, although the individual is unable to overtly detect these violations (Peretz, Brattico, Järvenpää, \& Tervaniemi, 2009). These data point to the pos- sibility that pitch sensitivity is normal in congenital amusia and that lack of confidence might be the origin of the observed behavioral failures. In normal participants, confidence and discriminability typically are related. However, in amusics, there might be a dissociation, as is the case in blindsight: Their discrimination abilities might be higher than what they think they are. Amusic individuals are often underconfident about their perceptual experiences. They may treat uncertainty as a lack of perception and report no awareness, even when more objective measures show that they can detect the presence of pitch changes.

Pitch perception without awareness raises the question of the nature of the neural representation that supports it. One possibility is that pitch-tracking mechanisms are normal up to the level of the auditory cortex, but the cortical neural representation of pitch is too weak to support reliable discrimination and memory. This would be consistent with the anatomical anomalies observed in the auditory and inferior frontal cortex of amusic individuals (Hyde, Zatorre, Griffiths, Lerch, \& Peretz, 2006; Hyde et al., 2007; Mandell, Schulze, \& Schlaug, 2007). Another possibility, as noted above, is that the auditory cortex computes pitch changes normally, but lack of confidence and decision factors are the origin of the observed behavioral failures. If this is the case, it should be possible to reveal normal sensitivity to pitch in indirect tasks that do not require overt detection of pitch changes. Revealing this sensitivity was the goal of the present study.

Here, we measured the influence of irrelevant pitch variations on time judgments by adapting a duration clas-

M.Pfeuty, micha.pfeuty@u-bordeaux2.fr 
sification task used by Grondin and Rousseau (1991), in which the listeners' ability to classify time intervals as "short" or "long" was shown to depend on the physical characteristics of the auditory signals bounding the intervals. When two auditory signals (a $1-\mathrm{kHz}$ tone, boundary $\mathrm{A}$, and white noise, boundary a) were used to signal the beginning and end of an otherwise silent time interval, listeners had a better performance for intervals bounded by identical auditory signals (AA and aa) than for intervals bounded by different auditory signals (Aa and aA). This was interpreted as the result of a timer that would take advantage of the repetition of an identical auditory signal. Here, we used the same type of task, but used tones that could vary on pitch as the auditory signals. We predicted that congenital amusics would have no difficulty in a duration classification task without pitch variations (Hyde \& Peretz, 2004). The key question was to what extent amusics' ability to classify empty intervals would be affected by the irrelevant pitch variations of the bounding tones that they are not aware of.

The notion that auditory perception depends on pitchtime interactions in a normal brain is well supported in the literature (e.g., Boltz, 1998; Crowder \& Neath, 1995; Henry \& McAuley, 2009; Lebrun-Guillaud \& Tillmann, 2007; Shigeno, 1993). The task that was used here was designed to create pitch-time interference with small pitch variations that amusics may be unaware of. To this aim, in Experiment 1, we assessed whether a quarter-semitone pitch difference (i.e., 25 cents) in the bounding tones was sufficient to affect performance in a duration classification task in a normal brain. The quarter-semitone difference was selected because it is detected easily by normal listeners, but not by amusic participants (Hyde \& Peretz, 2004). Since we found evidence of pitch interference effect on duration judgments in normal listeners, we tested 10 amusics and their matched controls in similar conditions (in Experiment 2). Our hypothesis was that irrelevant small pitch variations might influence temporal judgments in the amusic group because of the use of indirect/ implicit task requirements. However, we essentially failed to find support for this hypothesis. Therefore, in Experiment 3, we further tested amusics' ability to perform the same duration classification task, but with large pitch differences that they could reliably detect.

\section{EXPERIMENT 1}

\section{Method}

Participants. Twelve right-handed students ( 6 females, 6 males, 21-32 years of age), with no reported hearing deficit and no previous neurological or psychiatric history, participated in this experiment. None were musicians. They received a small compensation for their participation.

Procedure. The experiment consisted of a duration classification task made of two types of blocks: pitch-constant (PC) and pitchvarying $(\mathrm{PV})$.

Each block comprised an encoding phase and a comparison phase (Figure 1). During the encoding phase, a standard 200-msec interonset interval (IOI) made of two tones of identical pitch $(\mathrm{C} 6=1047 \mathrm{~Hz})$ was presented 6 times. Participants had to memorize this standard interval. During the comparison phase, two types of test intervals (short and long IOIs of 185 and $215 \mathrm{msec}$, respectively) were presented 28 times at random (14 short and 14 long IOIs). Participants had to judge the test interval as longer or shorter than the memorized standard. In the study by Grondin and Rousseau (1991), high scores of sensitivity $\left(d^{\prime}>3\right)$ were obtained with a similar difference. The encoding phase was repeated at the beginning of each block, so that participants could refresh their memories every 28 trials.

In PC blocks, all test intervals were bounded by two tones of identical pitch $(\mathrm{C} 6=1047 \mathrm{~Hz})$. In PV blocks, test intervals were bounded by seven possible pairings of tones separated by a maximum distance of a quarter-semitone. There were three intervals bounded by identical pitches (C6/C6, C61/C61, C6-/C6-) and four intervals bounded by different pitches (C6/C61, C61/C6, C6/C6-, C6-/C6), where C6 $=1047 \mathrm{~Hz}, \mathrm{C} 61=1062 \mathrm{~Hz}, \mathrm{C} 6-=1032 \mathrm{~Hz}$, with $\mathrm{C} 61$ and $\mathrm{C} 6-$ being 25 cents higher and lower than $\mathrm{C} 6$, respectively. We did not use the C6-/C61 and C61/C6- intervals, because in these pairings, the pitch distance is twice as large as the quarter-semitone considered here. The seven types of pairings were presented 28 times at random (4 per pairing) in each PV block.

There were six experimental blocks: three PC blocks and three PV blocks. In all, there were 84 trials ( 42 short and 42 long) per condition. In the PV condition, there were 12 trials $(6$ short and 6 long) for each pairing. Before the experimental blocks, participants performed a practice block of 14 trials with a feedback that consisted of the presentation of a word during $1 \mathrm{sec}$ just after the keypress: "correct" when they pressed the correct key, "incorrect" when they pressed the wrong key, and "late" when they did not give a response within a 2-sec delay. There was one practice block for the PC condition (practice PC) and another for the PV condition (practice PV). $\mathrm{PC}$ and PV blocks were presented in pairs, beginning with the pair of practice blocks, followed by the three pairs of blocks. The order was balanced: For half of the participants, each pair of blocks began with the PC condition, and for the other half, each pair of blocks began with the PV condition. Participants were not informed about the different types of blocks.

Stimuli and Apparatus. Tones were $100 \mathrm{msec}$ long and synthesized with a piano timbre, as was used in Hyde and Peretz (2004). They were delivered via headphones. Each participant adjusted the volume of the tones to a level that they considered to be comfortable. The volume was held constant throughout the experiment.

The task was presented on a PC via E-Prime, and participants used the keyboard to respond. Half of them had to press on the right key for the "shorter" response and the left key for the "longer" response. Key assignments were reversed for the other half. Participants were asked to respond as accurately as possible within a 2 -sec response window. The interval between the response and the next trial was $1.5 \mathrm{sec}$.

Data analysis. The main analyses in the present study were based on signal detection theory (Green \& Swets, 1966; Macmillan \& Creelman, 2005). Sensitivity $\left(d^{\prime}\right)$ was calculated as the difference between the z-score transformation of the hit and false alarm rates $\left[d^{\prime}=z(\mathrm{H})-z(\mathrm{FA})\right]$. A correct answer to a trial in which the test interval was long was considered as a hit. A false alarm was computed when a participant responded "long" in trials where the test interval was short. We also evaluated participants' bias in response criterion $(c)$, which is calculated as $-0.5 \times[z(\mathrm{H})+z(\mathrm{FA})]$, as defined in Macmillan and Creelman (2005). Response criterion was calculated in order to determine whether participants were more prone to respond "short" or "long." A criterion score of 0 would indicate that participants made an equivalent proportion of misses (i.e., responding "short" to a trial in which the test interval was long) and false alarms (i.e., responding "long" to a trial in which the test interval was short). A negative criterion score would indicate a bias to respond "long" (proportion of false alarms more important than proportion of misses), whereas a positive criterion score would indicate a bias to respond "short" (proportion of misses more important than proportion of false alarms).

The log-linear rule was applied to account for extreme cases where participants had a hit or false alarm rate of 0 or 1 , so that $N_{\mathrm{h}}=\left(N_{\mathrm{h}}+0.5\right) /(N+1)$ and $N_{\mathrm{f}}=\left(N_{\mathrm{f}}+0.5\right) /(N+1)$, where $N$ is 


\section{Pitch-Constant Blocks}

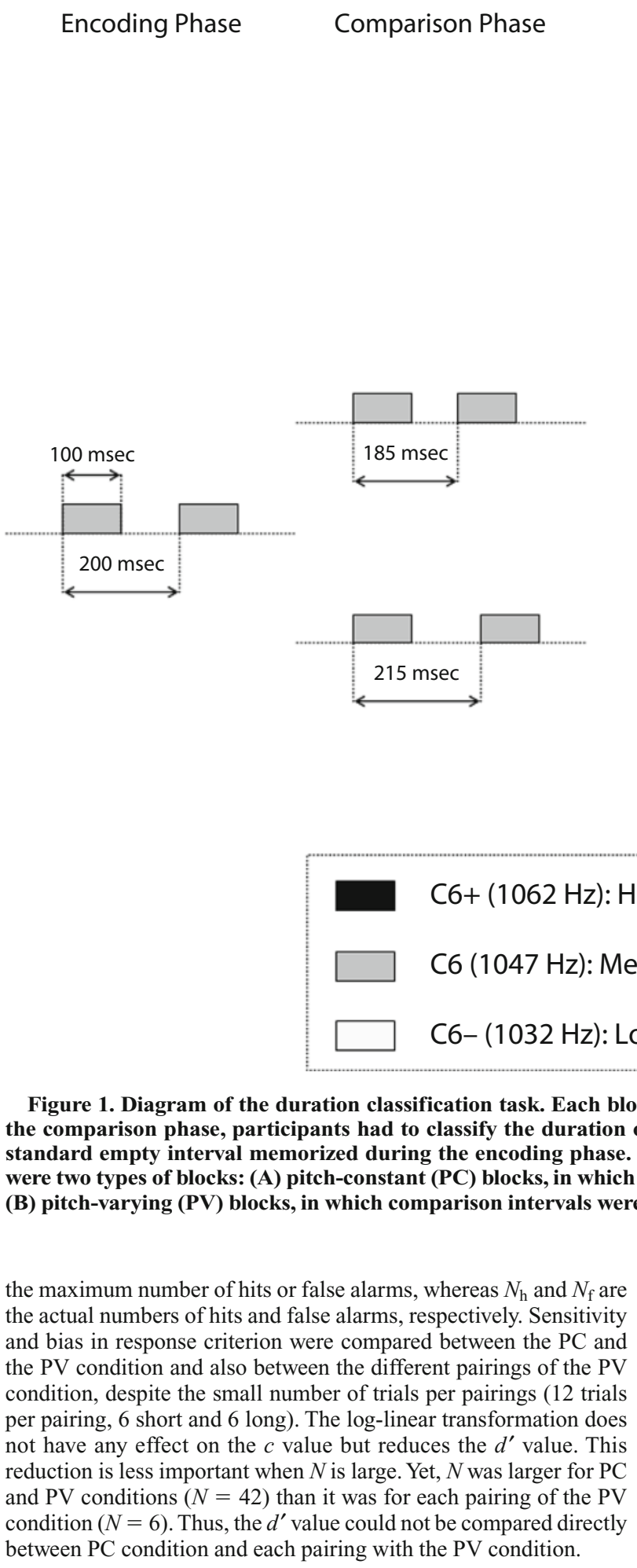

\section{Results}

Duration judgments were shown to have been impaired by the 25 -cent pitch changes, since the $d^{\prime}$ value was significantly lower in the PV $(M \pm S D, 1.41 \pm 0.79)$ than the maximum number of hits or false alarms, whereas $N_{\mathrm{h}}$ and $N_{\mathrm{f}}$ are the actual numbers of hits and false alarms, respectively. Sensitivity condition, despite the small number of trials per pairings (12 trials per pairing, 6 short and 6 long). The log-linear transformation does reduction is less important when $N$ is large. Yet, $N$ was larger for PC 列 condition $(N=6)$. Thus, the $d^{\prime}$ value could not be compared directl
between PC condition and each pairing with the PV condition.

\section{Pitch-Varying Blocks}

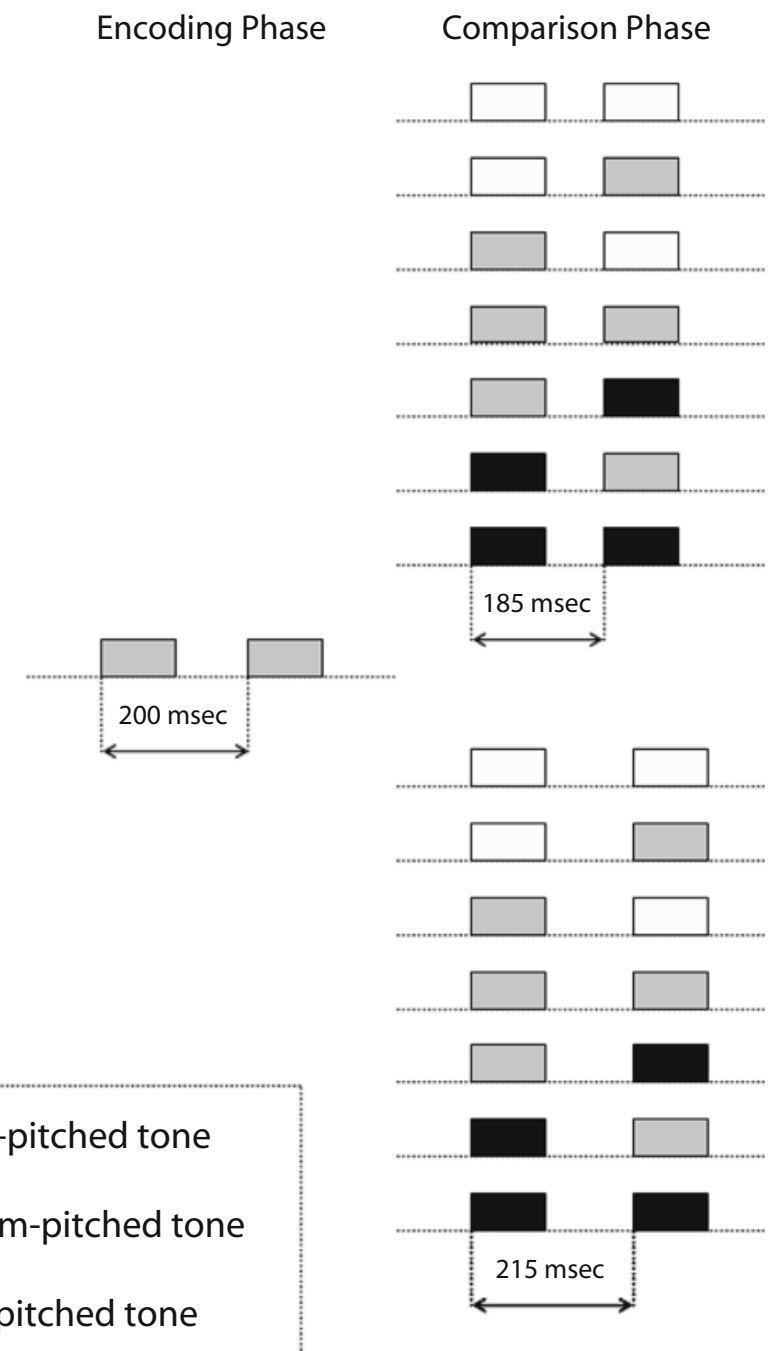

\begin{abstract}
Figure 1. Diagram of the duration classification task. Each block was composed of an encoding and a comparison phase. During the comparison phase, participants had to classify the duration of empty intervals as "shorter" or "longer" than the duration of a ( All encoding intervals were bounded by a single pitch (C6). There were two types of blocks: (A) pitch-constant (PC) blocks, in which all comparison intervals were bounded by the same pitch (C6), and
\end{abstract}

in the PC $[M \pm S D, 1.91 \pm 0.88 ; t(11)=3.10, p=.01]$ condition, as is shown in Figure 2A. Effect size was $d=$ 0.90, which was categorized by Cohen (1988) as a large effect. In the PV condition, there was no significant effect of pairing on the $d^{\prime}$ value $(F<1$; see Figure $2 \mathrm{~B})$. In particular, when $d^{\prime}$ values were pooled together for the three intervals bounded by identical pitches (low/low, medium/ medium, high/high) and for the four intervals bounded by different pitches (low/medium, medium/low, medium/ high, high/medium), there was no significant difference between intervals bounded by identical pitches and intervals bounded by different pitches $(F<1)$.

The $c$ value was significantly above 0 in the PC condition $[t(11)=3.07, p=.01]$, whereas it did not differ from 0 in the PV condition $(t<1)$. Furthermore, 

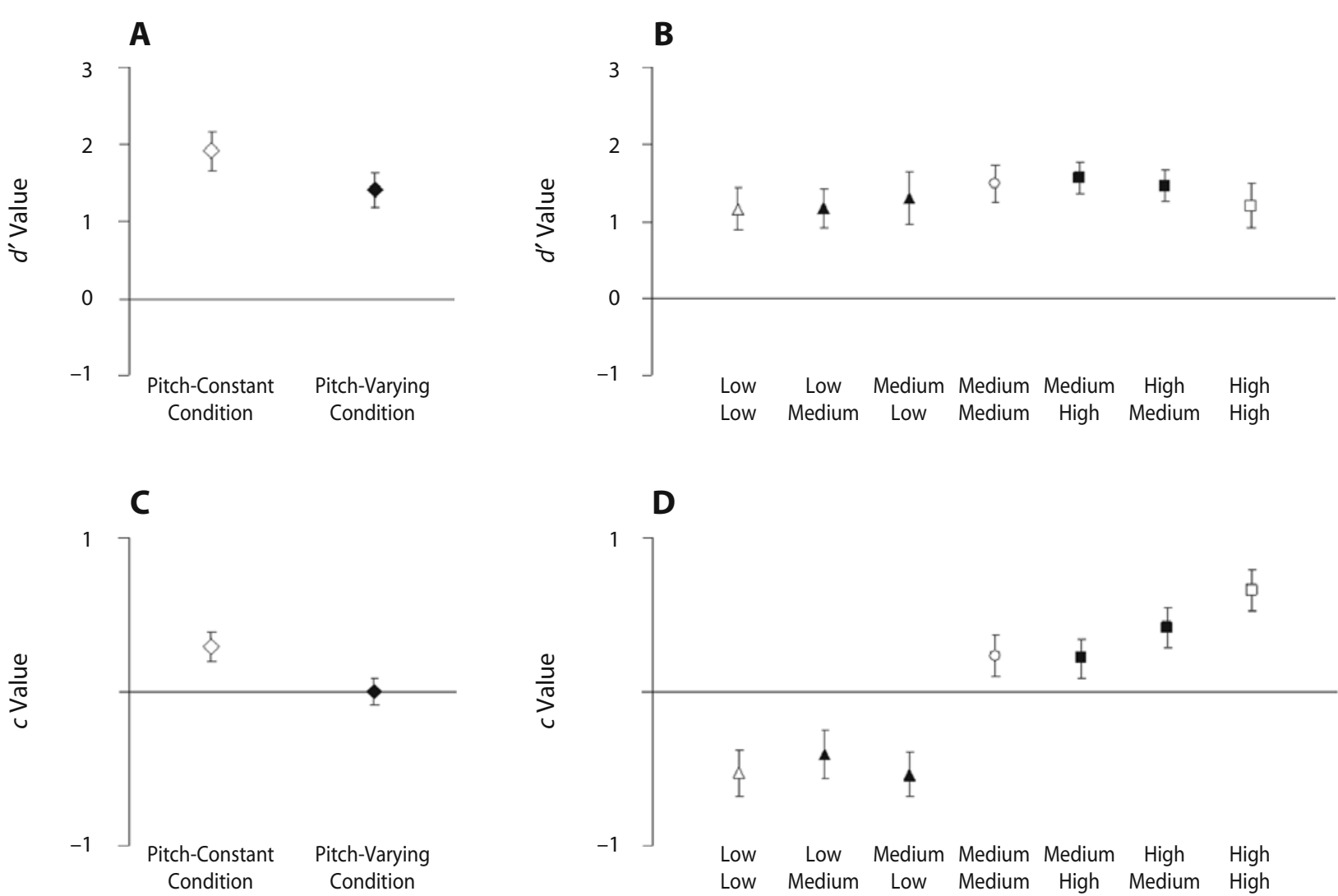

Figure 2. Effect of small pitch variations (a quarter of a semitone) on the performance of 12 normal participants in the duration classification task (Experiment 1). (A) Mean and standard error of the $d^{\prime}$ value in the pitch-constant (PC; empty diamond) and in pitch-varying (PV; filled diamond) conditions. (B) Mean and standard error of the $d^{\prime}$ value for the seven types of intervals presented in the PV condition. The three intervals bounded by identical pitches (low $/$ low, medium $/ \mathrm{medium}, \mathrm{high} / \mathrm{high}$ ) are represented by a white geometric shape, and the four intervals bounded by different pitches (low/medium, medium/low, medium/high, high/medium) are represented by a black geometric shape. The three intervals bounded by at least one low-pitched tone (low/low, low $/ \mathrm{medium}, \mathrm{medium} /$ low) are represented by a triangle, the three intervals bounded by at least one high-pitched tone (medium/high, high/medium, high/ high) are represented by a square, and the interval bounded by two medium-pitched tones ( $\mathrm{medium} / \mathrm{medium})$ is represented by a circle. (C) Mean and standard error of the $c$ value in the PC and PV conditions. (D) Mean and standard error of the $c$ value for the seven types of intervals presented in the PV condition.

it was higher in the $\mathrm{PC}(M \pm S D, 0.29 \pm 0.33)$ than in the PV $(M \pm S D, 0.00 \pm 0.31)$ condition $[t(11)=$ $4.63, p=.0007]$, with large effect size $(d=1.34)$; see Figure $2 \mathrm{C}$. In the $\mathrm{PV}$ condition, there was a significant effect of pairing on the $c$ value $\left[F(6,66)=15.14, M S_{e}=\right.$ $\left.12.60, p<.0001, \eta^{2}=.58\right]$; see Figure 2D. The $c$ value was higher for the three intervals bounded by identical pitches $(M \pm S D, 0.12 \pm 0.24)$ than for the four intervals bounded by different pitches $[M \pm S D,-0.08 \pm 0.34$; $t(11)=2.38, p=.04]$. The effect size was medium $(d=$ 0.69 ). The $c$ value was also higher for the three pairings bounded by at least one high-pitched tone (medium/high, $\mathrm{high} /$ medium, high/high; $M \pm S D, 0.43 \pm 0.39$ ) than for the three pairings bounded by at least one low-pitched tone [low/low, low/medium, medium/low; $M \pm S D$, $-0.49 \pm 0.41 ; t(11)=5.64, p=.0002]$. The effect size was large $(d=1.63)$. Finally, it is of note that the $c$ value for the medium-medium pairing $(M \pm S D, 0.23 \pm 0.46)$ was very close to that for the PC condition $(M \pm S D$, $0.29 \pm 0.33)$.

\section{Discussion}

Sensitivity was lower in the PV than in the PC condition, indicating that quarter-semitone pitch variations are sufficient to affect accuracy in duration classification. However, the PV condition differed from the PC condition in two aspects: First, test intervals were bounded by seven possible pairings in the PV condition (three intervals bounded by identical pitches and four intervals bounded by different pitches). Second, all pairings were presented randomly in each block, and it was not possible for participants to predict which pairing of tones would appear. The lower sensitivity observed in the PV condition compared with the PC condition could be due either to some of the test intervals (in particular to the intervals bounded by different pitches) or to pitch uncertainty (due to the randomized presentation). The fact that, in the PV condition, sensitivity was not higher for intervals bounded by identical pitches than for intervals bounded by different pitches gives support to the latter hypothesis. The lower sensitivity observed in the PV condition compared with the PC 
condition would be due to pitch uncertainty. This is in line with several studies showing that temporal judgments are affected by randomizing marker parameters (Grondin \& Rousseau, 1991; Penner, 1976).

The propensity to respond "long" was more important when there were irrelevant pitch variations (in the PV condition) than when there were none (in the PC condition). Furthermore, in the PV condition, the propensity to respond "long" was more important for trials containing different pitches than for trials made of identical pitches. This indicates that intervals bounded by different pitches were judged as longer than intervals bounded by identical pitches. Using a similar task, Grondin and Rousseau (1991) reported a similar tendency to respond "long" more often for intervals bounded by different tones than for those bounded by identical tones. This effect is consistent with the view that duration classification of empty intervals relies on a pacemaker that provides pulses counted from the offset of the first marker to the onset of the second marker. It is likely that the second marker is detected earlier by the auditory system when it is identical to the first marker due to repetition priming. Accordingly, the time period during which the pacemaker emits pulses would be reduced, leading to a shorter temporal judgment.

This effect of pitch difference on duration classification is also consistent with the auditory kappa effect. The kappa effect initially was demonstrated for visual stimuli (Cohen, Hansel, \& Sylvester, 1953). The classical task involves the successive presentation of three light flashes, which indicates two different intervals (of distance and duration). A longer spatial distance interval between two flashes leads to the interval being perceived as longer in duration. Cohen, Hansel, and Sylvester (1954) considered the possibility of an auditory kappa effect in which the three flashes of light are replaced by tones and spatial distance by pitch distance. Unfortunately, they failed to obtain a significant effect. However, more recent studies using different procedures observed that perceived duration between two tones is lengthened when the pitch separation increases (Boltz, 1998; Crowder \& Neath, 1995; Henry \& McAuley, 2009; Shigeno, 1993). In line with this, we showed that the perceived duration was longer for a pitch separation of 25 cents than when there was no pitch separation.

The propensity to respond "long" was also more important for intervals bounded by at least one low-pitched tone than for intervals bounded by at least one high-pitched tone. This effect of pitch level was much larger than the effect of pitch difference. Previous studies have revealed that pitch level affects the perceived duration of a continuous tone. Cohen et al. (1954) also observed that, when participants have to adjust the duration of a high-pitched tone with the duration of a low-pitched tone, the time allotted to the higher tone is less than that allotted to the lower tone. The high-pitched tone would thus be perceived as longer than the low-pitched tone. A similar result was obtained by Brigner (1988). However, to our knowledge, no study revealed an effect of markers' pitch level on the perception of an empty interval.
A first possible account is that this effect would result from the influence of markers' perceived duration. Previous studies have shown that a continuous tone is perceived as longer when its frequency is higher (Brigner, 1988; Cohen et al., 1954). Yet empty intervals tend to be overestimated in duration when they are bounded by longer markers (Grondin, Ivry, Franz, Perreault, \& Metthé, 1996). Thus, intervals bounded by high-pitched tones should be perceived as longer than intervals bounded by low-pitched tones. This is the opposite of what we observed here. However, it is possible that, in our study, participants estimated for each interval the ratio between the empty and the filled period to make their temporal judgment. In this case, if low-pitched tones are perceived to be shorter, the ratio between the empty and the filled period should be higher, leading to a "long" response.

Another possible account of the association between pitch and duration is that high/low pitches are associated to small/large sizes, which, in turn, are associated to short/ long durations. It has been observed that a visual stimulus composed of an array of dots is more likely to be judged as "long" when it contains more, larger, or brighter dots (Xuan, Zhang, He, \& Chen, 2007). Similarly, the duration of a digit is perceived as being longer for high than for low digits (Oliveri et al., 2008). Finally, empty intervals have also been shown to last subjectively longer when they are bounded by large rather than small markers (Ono \& Kitazawa, 2009). According to Ivry and Schlerf (2008), these illusions would result from associations between spatial and temporal concepts, which would influence temporal judgments at decision level. Few and many or small and large would be associated with short and long, respectively. In parallel, Ohala $(1983,1984)$ proposed the concept of frequency code, according to which highand low-pitched vocalizations inform about smallness and largeness of the vocalizer. In line with this concept, Stevens (1934) observed that, when observers are asked to make two tones of different frequency sound equal in volume (largeness), they increase the intensity of the higher tone, thereby indicating that the lower tones sound "larger." Accordingly, we propose that high/low pitches are associated to small/large sizes, which, in turn, would be associated to short/long durations. In other words, the greater tendency to respond "long" for intervals bounded by low-pitched tones than for intervals bounded by highpitched tones would result from an association between pitch level and duration. However, this hypothesis contradicts the fact that a continuous tone is perceived as longer when its frequency is higher (Brigner, 1988; Cohen et al., 1954). More investigations are needed for a better understanding of the influence of pitch level on temporal judgment.

Interestingly, the same type of association has been reported between pitch and spatial location (Lidji, Kolinsky, Lochy, \& Morais, 2007; Rusconi, Kwan, Giordano, Umiltà, \& Butterworth, 2006), referring to the SMARC (spatialmusical association of response codes) effect. Timbre judgments are typically made more quickly when participants respond to high-frequency tones with a key located high 
in space and to low-frequency tones with a spatially lower key than when the relationship is reversed. This spatial representation of pitch is consistent with the Western cultural norm in which high-frequency tones are usually represented as higher in space than lower frequency tones (Pratt, 1930). Biological factors could also account for this pitch-space association, since, for example, the larynx is moved higher in the throat to produce higher pitch tones. The present pitch-duration association between high- and low-pitched tones and short/long durations may reflect similar regularities as those found for pitch-space relations.

In sum, we showed here that irrelevant small pitch variations influenced the performance of normal participants in a duration classification task. The situation was thus deemed appropriate to investigate indirect pitch processing in congenital amusia.

\section{EXPERIMENT 2}

\section{Method}

Participants. The participants were 10 amusics and 10 matched controls, as summarized in Table 1. Each amusic individual was matched in age, education, and musical background to a participant with no musical problems and no formal musical education. None of the participants had any previous neurological or psychiatric history. Objective testing confirmed the presence of a musical deficiency in the amusic participants, who scored significantly below the matched control participants in each test taken from the Montreal Battery of Evaluation of Amusia (MBEA; Peretz, Champod, \& Hyde, 2003). The battery involves six tests - three to assess the ability to discriminate changes in melody (by pitch contour, scale, and interval size) and one to assess rhythmic discrimination (by temporal grouping).

Stimuli, Apparatus, and Procedure. The experiment consisted of a duration classification task followed by a control pitch direction task.

Duration classification task. The duration classification task was the same as that of Experiment 1, except that different test durations were used here. Since amusic and control participants are much older than students and timing performance decreases with aging (Wearden, Wearden, \& Rabbitt, 1997), the separation between standard and test durations was increased from $7.5 \%(15 \mathrm{msec})$ to $10 \%$ $(20 \mathrm{msec})$. Thus, the short and long test durations were set to 180 and $220 \mathrm{msec}$, respectively.

Pitch direction task. The pitch direction task was added to ascertain that the 25-cent pitch variations used in the duration classification task were perceived by controls, and not by amusics when tested explicitly (e.g., Loui et al., 2008). Four pairs of different pitches (C6/ $\mathrm{C} 6+, \mathrm{C} 6+/ \mathrm{C} 6, \mathrm{C} 6 / \mathrm{C} 6-$, and $\mathrm{C} 6-/ \mathrm{C} 6)$ were presented 48 times at random in a single block. For each pair, participants had to determine whether the second tone was "higher" or "lower" in pitch than the first one. The IOIs were 180 or $220 \mathrm{msec}$, as in the duration discrimination task. Before the beginning of the task, participants performed one practice session of 16 trials with a feedback that consisted of the presentation of a word during $1 \mathrm{sec}$ just after the keypress: "correct" when they pressed the correct key, "incorrect" when they pressed the wrong key, and "late" when they did not give response in the 2-sec delay. The task was presented on a PC computer via E-Prime, and participants used the keyboard to respond. Half of them had to press on the right key for the "higher" response and the left key for the "lower" response. Keys were inverted for the second half. Participants were asked to respond as accurately as possible in a response time window of $2 \mathrm{sec}$. The interval time between the response and the next trial was $1.5 \mathrm{sec}$

\section{Results}

In order to determine whether a pitch interference effect on duration judgments was present within each group, analyses were first performed separately. In subsequent analyses, we tested the effect of the group (control vs. amusic). Finally, correlation analyses were performed in each group between explicit and implicit measures of pitch processing and the MBEA score.

Control group. In the pitch direction task, control participants were able to perceive 25 -cent pitch changes better than chance since the $d^{\prime}$ value was significantly above zero $[M \pm S D, 1.87 \pm 1.49 ; t(9)=3.96, p=.003]$. The effect size was large $(d=1.25)$.

In the duration classification task, duration judgments were impaired by small and irrelevant pitch variations since the $d^{\prime}$ value was lower in the PV condition $(M \pm S D, 1.36 \pm 0.88)$ than in the $\mathrm{PC}$ condition $(M \pm S D$, $1.97 \pm 1.06)[t(9)=4.14, p=.003$; Figure $3 \mathrm{~A}]$ with a large effect size $(d=1.31)$. In the PV condition, there was no significant effect of pairing on the $d^{\prime}$ value $(F<1$; Figure 3B).

The $c$ value was not significantly different from zero in either the PC or PV condition $(t<1)$ and did not significantly differ between the two conditions $(t<1$; see Figure $3 \mathrm{C})$. In the $\mathrm{PV}$ condition, there was a significant effect of pairing on the $c$ value $\left[F(6,54)=6.61, M S_{e}=0.18\right.$, $p<.0001, \eta^{2}=.43$; Figure 3D], which was higher for the three pairings bounded by at least one high-pitched tone $(M \pm S D, 0.30 \pm 0.34)$ than for the three pairings bounded by at least one low-pitched tone $[M \pm S D,-0.33 \pm 0.36$;

Table 1

Characteristics of the Participants and Mean Percentage of Correct Responses on Montreal Battery of Evaluation of Amusia (MBEA; Peretz, Champod, \& Hyde, 2003) Tests

\begin{tabular}{|c|c|c|c|c|c|}
\hline \multirow[b]{3}{*}{ Characteristic } & \multicolumn{4}{|c|}{ Group } & \multirow[b]{3}{*}{$t$ Test } \\
\hline & \multicolumn{2}{|c|}{ Amusic $(n=10)$} & \multicolumn{2}{|c|}{ Control $(n=10)$} & \\
\hline & M & $S E$ & $M$ & $S E$ & \\
\hline Age (years) & 60.2 & 3.1 & 59.4 & 2.2 & n.s. \\
\hline Education (years) & 17.1 & 0.8 & 16.7 & 0.4 & n.s. \\
\hline MBEA global score & 65.5 & 2.4 & 88.9 & 1.1 & $t(18)=8.76, p<.0001$ \\
\hline MBEA melodic test scores & 61.6 & 2.2 & 88.9 & 1.8 & $t(18)=9.54, p<.0001$ \\
\hline MBEA rhythmic test scores & 77.0 & 4.6 & 88.7 & 2.4 & $t(18)=2.25, p<.05$ \\
\hline
\end{tabular}

Note-Gender for the amusic group: 7 female, 3 male; gender for the control group: 6 female, 4 male. 
A

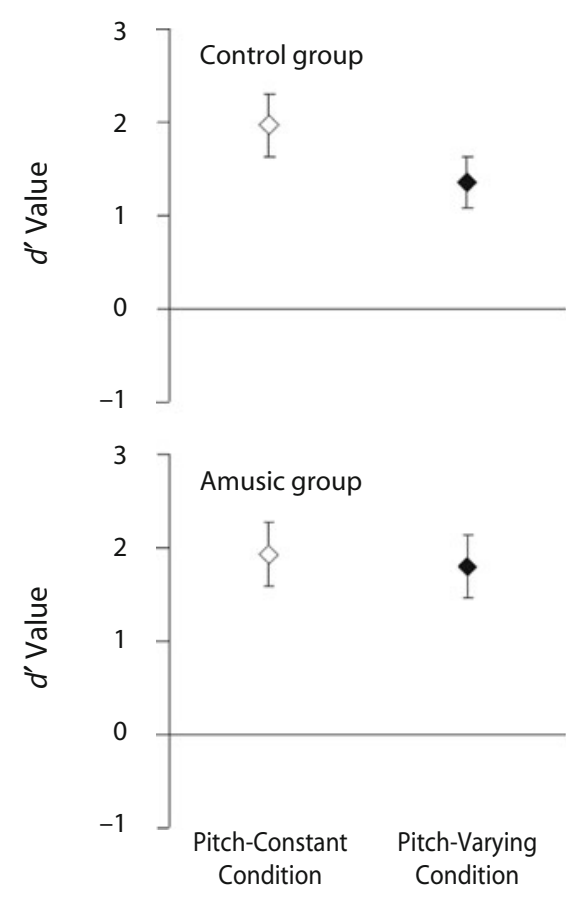

C

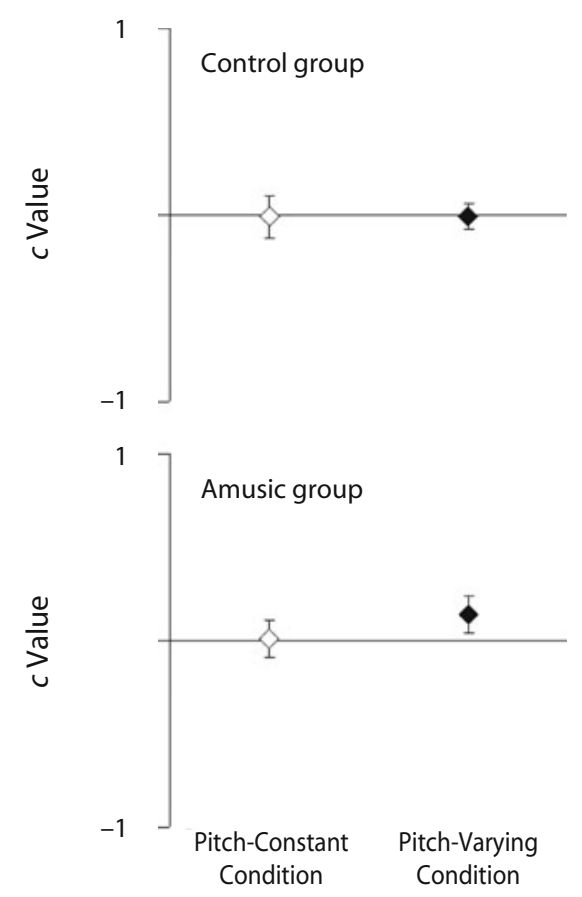

B

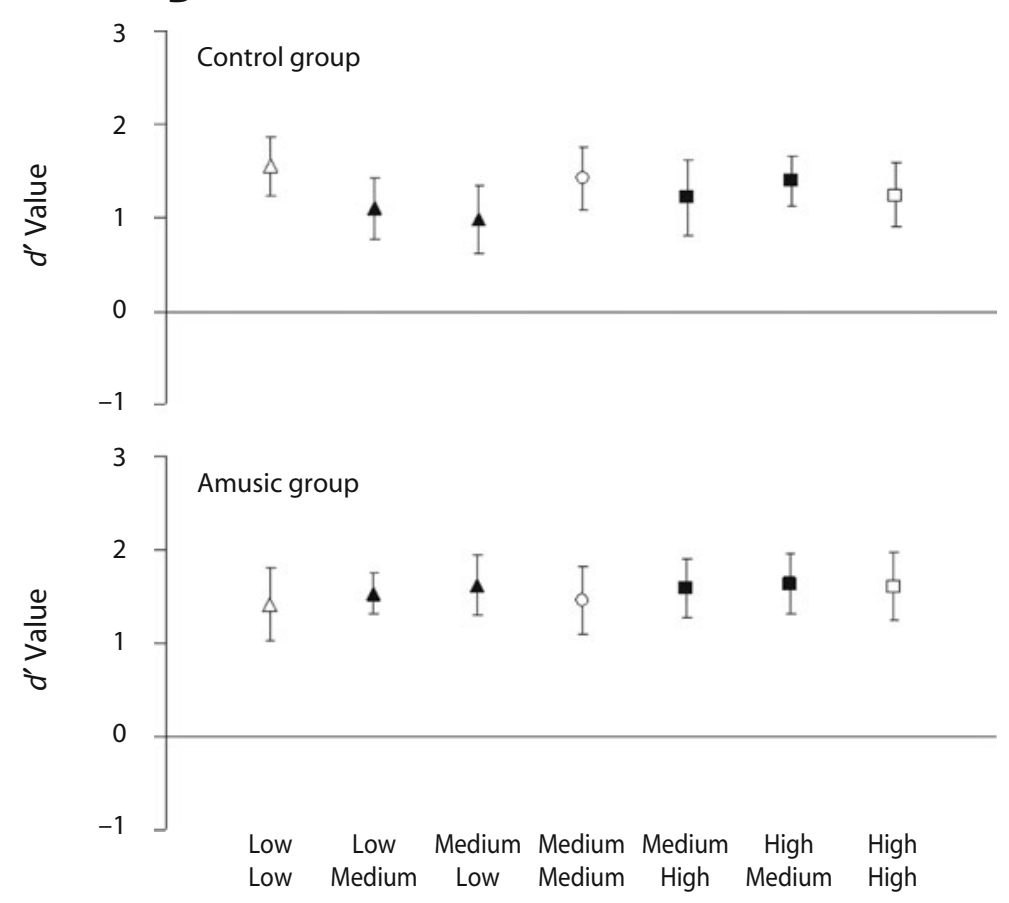

D
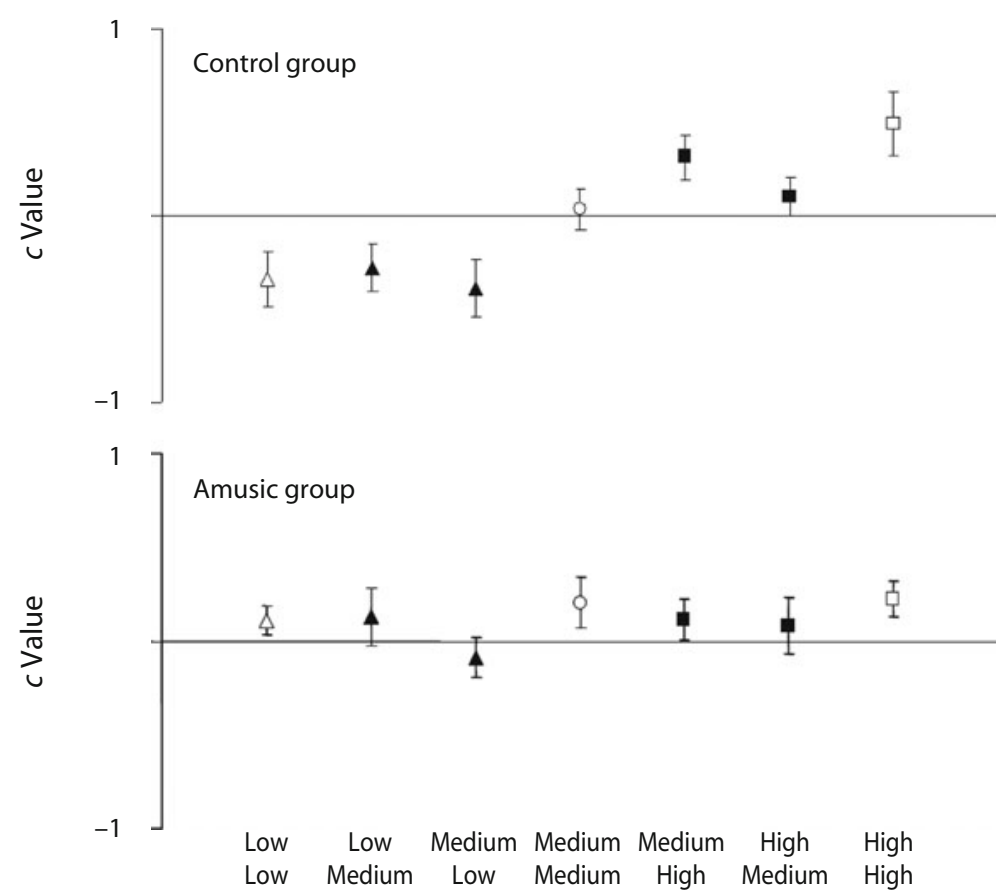

Figure 3. Effect of small pitch variations (a quarter of a semitone) on the performance of 10 control and 10 amusic participants in the duration classification task (Experiment 2). (A) Mean and standard error of the $d^{\prime}$ value in the pitch-constant (PC) and pitch-varying (PV) conditions for the control (at the top) and the amusic (at the bottom) groups. (B) Mean and standard error of the $d^{\prime}$ value for the seven types of intervals presented in the PV condition for the control (at the top) and the amusic (at the bottom) groups. (C) Mean and standard error of the $c$ value in the PC and in the PV condition for the control (at the top) and the amusic (at the bottom) groups. (D) Mean and standard error of the $c$ value for the seven types of intervals presented in the PV condition for the control (at the top) and the amusic (at the bottom) groups. 
$t(9)=3.41, p=.008]$, with a large effect size $(d=1.08)$. There was no significant difference between intervals bounded by identical pitches and intervals bounded by different pitches $[t(9)=1.25, p=.24]$.

Amusic group. The amusic participants were unable to differentiate pitch direction. Their performance was at chance $\left[d^{\prime}\right.$ value: $M \pm S D, 0.17 \pm 0.40 ; t(9)=1.35, p=$ $.21]$, with a small effect size $(d=0.43)$.

Duration judgments were not impaired by irrelevant pitch variations since the $d^{\prime}$ value was not significantly lower in the PV $(M \pm S D, 1.80 \pm 1.07)$ than in the PC $(M \pm S D, 1.93 \pm 1.07)$ condition $(t<1$; Figure $3 \mathrm{~A})$, with a small effect size $(d=0.22)$. In the PV condition, there was no significant effect of pairing on the $d^{\prime}$ value $(F<1$; Figure 3B).

The $c$ value was not significantly different from zero in either the PC $(t<1)$ or PV $[t(9)=1.42, p=.19]$ condition and did not significantly differ between the two conditions $[t(9)=1.55, p=.16]$, as shown in Figure 3C. In the PV condition, there was no significant effect of pairing on the $c$ value $(F<1$; Figure 3D).

Amusic vs. control comparison. As expected, controls performed much better than amusics in the pitch direction task $\left[d^{\prime}\right.$ value: $M \pm S D$, controls: $1.87 \pm 1.49$, amusics: $0.17 \pm 0.40 ; t(18)=3.48, p=.003]$, with a large effect size $(d=1.64)$. In the duration classification task, an ANOVA was carried out on the $d^{\prime}$ value with condition (PC vs. PV) and group (control vs. amusic) as factors. There was no effect of group $(F<1)$, an effect of condition $\left[F(1,18)=9.54, M S_{e}=0.15, p=.006, \eta^{2}=\right.$ .35 , and the interaction between group and condition approached significance $\left[F(1,18)=4.00, M S_{e}=0.15, p=\right.$ $\left..06, \eta^{2}=.18\right]$. In order to evaluate the interference effect created by the presence of irrelevant pitch variations at an individual level, we subtracted the $d^{\prime}$ value in the PV condition from that in the PC condition, giving a $d^{\prime}{ }_{\mathrm{PC}}-\mathrm{PV}$ value. This $d^{\prime}{ }_{\mathrm{PC}}-\mathrm{pV}$ value tended to be higher for control $(M \pm S D, 0.61 \pm 0.47)$ than for amusic $(M \pm S D$, $0.13 \pm 0.60)$ participants $[t(18)=1.98, p=.06]$, with a relatively large effect size $(d=0.93)$.

In the PV condition, a 2 (group) $\times 7$ (pairing) ANOVA computed on the $c$ value revealed no effect of group $\left[F(1,18)=1.85, M S_{e}=0.26, p=.19, \eta^{2}=.09\right]$, an effect of pairing $\left[F(6,108)=5.68, M S_{e}=0.15, p<.0001\right.$, $\left.\eta^{2}=.24\right]$, and an interaction between group and pairing $\left[F(6,108)=2.79, M S_{e}=0.15, p=.01, \eta^{2}=.13\right]$. This reflected the fact that controls', but not amusics', duration judgments were affected by pitch level. In order to evaluate a pitch-level effect on duration judgments at an individual level, we subtracted the $c$ value averaged for intervals bounded by at least one high-pitched tone from the $c$ value averaged for intervals bounded by at least one lowpitched tone, giving a $c_{\text {High }- \text { Low }}$ value. This $c_{\text {High - Low }}$ value was higher for control $(M \pm S D, 0.64 \pm 0.59)$ than for amusic $[M \pm S D, 0.09 \pm 0.30]$ participants $[t(18)=$ $2.61, p=.02]$, with a large effect size $(d=1.23)$.

Correlation analyses. To assess potential associations between explicit and implicit measures of pitch processing and the MBEA score, we calculated nonparametric Spearman rank-order correlation coefficients for each group (Table 2). The $d^{\prime}$ PitchDir value (sensitivity in the pitch direction task) was taken as explicit measure. The $d_{\mathrm{PC}-\mathrm{PV}}^{\prime}$ value (interference effect created by irrelevant pitch variations on duration judgments) and the $c_{\text {High - Low }}$ value (pitch-level effect on duration judgments) were taken as implicit measures.

In the amusic group, the $c_{\text {High - Low }}$ value was correlated positively with the $d^{\prime}$ PitchDir value and with the MBEA score. Thus, implicit and explicit detection of small pitch changes appear to be related. Marginally significant positive correlations were also observed between the $c_{\text {High - Low }}$ value and the $d^{\prime}{ }_{\mathrm{PC}}-\mathrm{PV}$ value, between the $d^{\prime}{ }_{\mathrm{PitchDir}}$ value and the MBEA score, and between the $d^{\prime}{ }_{\mathrm{PC}}-\mathrm{PV}$ value and the MBEA score. In the control group, the $c_{\mathrm{High}}$ - Low value was correlated positively with the $d^{\prime}$ PitchDir value. Marginally significant positive correlations were observed between the $c_{\text {High - Low }}$ value and the MBEA score and between the $d^{\prime}$ PitchDir value and the MBEA score. However, none of these correlations was significant when Bonferroni's correction for multiple comparisons was applied.

\section{Discussion}

The older control participants tested here showed both covert and overt sensitivity to quarter-semitone pitch variations, as did students in Experiment 1. They succeeded in judging the direction of these pitch changes. Moreover, the quarter-semitone differences interfered with their duration judgments. Actually, sensitivity was lower in the PV condition than in the PC condition, probably as the result of a global pitch-uncertainty effect. Furthermore, control participants showed the association between high/low pitch tones and short/long durations. However, contrary

Table 2

Nonparametric Spearman Rank-Order Correlation Coefficients, $r_{\mathrm{s}}(d f)$, Across the $d^{\prime}$ PitchDir Value, the $d^{\prime}{ }_{\mathrm{PC}-\mathrm{PV}}$ Value, the $c_{\mathrm{Hig}}$. Value, and the Montreal Battery of Evaluation of Amusia (MBEA; Peretz, Champod, \& Hyde, 2003) Scores

\begin{tabular}{|c|c|c|c|c|c|c|c|c|c|c|c|c|}
\hline & \multicolumn{12}{|c|}{ Group } \\
\hline & \multicolumn{6}{|c|}{ Amusic $(n=10)$} & \multicolumn{6}{|c|}{ Control $(n=10)$} \\
\hline & \multicolumn{2}{|c|}{$d_{\text {PitchDir }}^{\prime}$} & \multicolumn{2}{|c|}{$d_{\mathrm{PC}-\mathrm{PV}}^{\prime}$} & \multicolumn{2}{|c|}{$c_{\text {High }- \text { Low }}$} & \multicolumn{2}{|c|}{$d_{\text {PitchDir }}^{\prime}$} & \multicolumn{2}{|c|}{$d_{\mathrm{PC}-\mathrm{PV}}^{\prime}$} & \multicolumn{2}{|c|}{$c_{\text {High }- \text { Low }}$} \\
\hline & $r_{\mathrm{s}}(8)$ & $p$ & $r_{\mathrm{s}}(8)$ & $p$ & $r_{\mathrm{s}}(8)$ & $p$ & $r_{\mathrm{s}}(8)$ & $p$ & $r_{\mathrm{s}}(8)$ & $p$ & $r_{\mathrm{s}}(8)$ & $p$ \\
\hline $\begin{array}{l}\text { MBEA score } \\
d_{\text {PitchDir }}^{\prime} \\
d_{\text {PC - PV }}^{\prime}\end{array}$ & 0.61 & .06 & $\begin{array}{l}0.62 \\
0.05\end{array}$ & $\begin{array}{l}.06 \\
.88\end{array}$ & $\begin{array}{l}0.76 \\
0.67 \\
0.59\end{array}$ & $\begin{array}{l}.01^{*} \\
.03^{*} \\
.07\end{array}$ & 0.62 & .06 & $\begin{array}{r}0.19 \\
-0.11\end{array}$ & $\begin{array}{l}.60 \\
.76\end{array}$ & $\begin{array}{r}0.62 \\
0.67 \\
-0.06\end{array}$ & $\begin{array}{l}.06 \\
.03^{*} \\
.85\end{array}$ \\
\hline
\end{tabular}


to students of Experiment 1, there was no evidence of extension of the perceived duration for intervals bounded by different pitches.

Unlike the matched controls, the amusic participants showed little sensitivity to the quarter-semitone variations, both overtly in pitch direction judgments and covertly in duration judgments. In particular, their performance in the duration classification task did show a decrement in the PV condition as compared with the PC condition. This suggests that amusic participants were not sensitive to the global pitch variability. A likely explanation is that the different pitches presented during the PV condition were not perceived as sufficiently different by amusics to create uncertainty. These findings support the view that the pitch deficit experienced by amusic individuals is not related to decision factors. Their behavioral failures observed in explicit pitch discrimination tasks (Foxton et al., 2004; Hyde \& Peretz, 2004) would result from a poor pitch resolution system rather than from lack of confidence.

Furthermore, the global performance of amusics in the duration classification task was comparable with that of controls. This result is in line with the psychophysical study of Hyde and Peretz (2004), showing that amusic individuals are impaired in pitch, but not in time, processing.

Interestingly, in the amusic group, the MBEA score was, or tended to be, correlated with both explicit and implicit measures of pitch processing (when no correction for multiple comparisons was applied). In other words, the less severe cases may show both overt and covert sensitivity to the quarter-semitone pitch variations, whereas the most severe would fail on both. Furthermore, the pitchlevel effect on duration judgments was positively correlated with sensitivity in the pitch direction task in both amusic and control groups. Thus, individuals with higher ability to discriminate pitch direction were more prone to associate high- and low-pitched tones with short and long durations, respectively.

In sum, our results show that, for quarter-semitone pitch variations, pitch-time interference is present in control but not in amusic participants. This strongly suggests that the pitch defect experienced by amusics is perceptual. Alternatively, the pitch defect could be due to a more general lack of sensitivity to pitch-time dependencies. In particular, the lack of global pitch-uncertainty effect observed in amusics could be explained by a memory deficit rather than a perceptual deficit. According to this memory account, amusics would have perceived the different pitches in the PV condition accurately but would have failed to memorize these pitches from one trial to another. If this is correct, no pitch-time interference effect should be observed in amusics when large pitch variations are used. Experiment 3 was designed to test this possibility.

\section{EXPERIMENT 3}

\section{Method}

Nine adult amusics who had participated in Experiment 2 performed the same duration classification task, except that, in the $\mathrm{PV}$ condition, the bounding pitches, $\mathrm{C} 6+=1062 \mathrm{~Hz}$ and $\mathrm{C} 6-=$ $1032 \mathrm{~Hz}$, were replaced by C $6++=1304 \mathrm{~Hz}$ and C6 $--=790 \mathrm{~Hz}$, which are 4 semitones higher and lower than C6, respectively. No pitch direction task was performed, because pitch changes of 4 semitones fall within the pitch discrimination abilities of amusics (Hyde \& Peretz, 2004).

\section{Results}

Here, amusics performed better in the duration classification when pitch was constant than when pitch was varied [ $d^{\prime}$ value: $M \pm S D$; $\mathrm{PC}$ condition: $1.39 \pm 0.88, \mathrm{PV}$ condition: $0.65 \pm 0.53 ; t(9)=3.96, p=.004]$, as shown in Figure $4 \mathrm{~A}$, with a large effect size $(d=1.32)$. In the PV condition, there was no significant effect of pairing on the $d^{\prime}$ value $\left[F(6,48)=1.65, M S_{e}=0.43, p=.15, \eta^{2}=.17\right]$, as shown in Figure 4B.

The $c$ value was significantly above zero in both the $\mathrm{PC}[M \pm S D, 0.43 \pm 0.48 ; t(8)=2.67, p=.03]$ and the $\mathrm{PV}[M \pm S D, 0.34 \pm 0.21 ; t(8)=5.00, p=.001]$ conditions, with a large effect size in both cases $(d=0.89$ and $d=1.67$, respectively), but it did not differ between the two conditions $(t<1$; Figure $4 \mathrm{C})$. In the PV condition, there was a marginally significant effect of pairing on the $c$ value $\left[F(6,48)=1.99, M S_{e}=0.34, p=.08\right.$, $\eta^{2}=.20$; Figure 4D]. The $c$ value was higher for intervals bounded by identical $(M \pm S D, 0.57 \pm 0.46)$ than for intervals bounded by different $(M \pm S D, 0.16 \pm 0.16)$ pitches $[t(8)=2.40, p=.04]$, with a large effect size $(d=0.80)$. However, contrary to what was found in normal participants in Experiments 1 and 2, the $c$ value was not significantly higher for the three pairings bounded by at least one high-pitched tone $(M \pm S D, 0.46 \pm 0.44)$ than for the three pairings bounded by at least one low-pitched tone $[M \pm S D, 0.13 \pm 0.36 ; t(8)=1.51, p=.17]$.

We also compared sensitivity between the PC and the PV condition and across experiments (Experiment 2 vs. Experiment 3). The 2 (experiment) $\times 2$ (condition) ANOVA yielded a main effect of experiment $[F(1,8)=$ 17.37, $\left.M S_{e}=0.32, p=.003, \eta^{2}=.69\right]$ and a main effect of condition $\left[F(1,8)=12.99, M S_{e}=0.13, p=.007, \eta^{2}=\right.$ $.62]$, and the interaction between experiment and condition approached significance $\left[F(1,8)=3.68, M S_{e}=0.23\right.$, $\left.p=.09, \eta^{2}=.32\right]$. These results support the observation that amusics' performance in the duration classification task decreased between Experiment 2 and Experiment 3 in the PV condition $\left[d^{\prime}\right.$ value: $M \pm S D$, Experiment 2 : $1.73 \pm 1.11$, Experiment 3: $0.65 \pm 0.53 ; t(8)=4.45$, $p=.002]$, but not in the PC condition [ $d^{\prime}$ value: $M \pm S D$, Experiment 2: $1.86 \pm 1.11$, Experiment 3: $1.39 \pm 0.88$; $t(8)=1.91, p=.09]$.

\section{Discussion}

Here, we show that amusics' duration judgments are affected by large pitch variations. This suggests that the lack of effect of pitch variations on duration classification observed in Experiment 2 was not due to task factors or to a memory deficit but arose from a deficit in perceiving fine-grained pitch differences.

In line with the results of Experiment 1, the propensity to respond "long" in the PV condition was more important for intervals bounded by different pitches than for intervals bounded by identical pitches, indicating an extension 
A

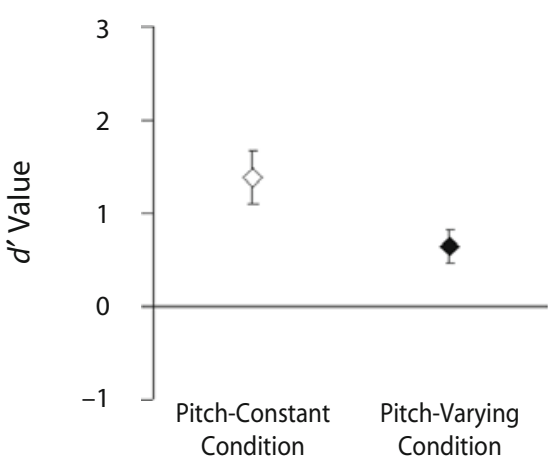

C

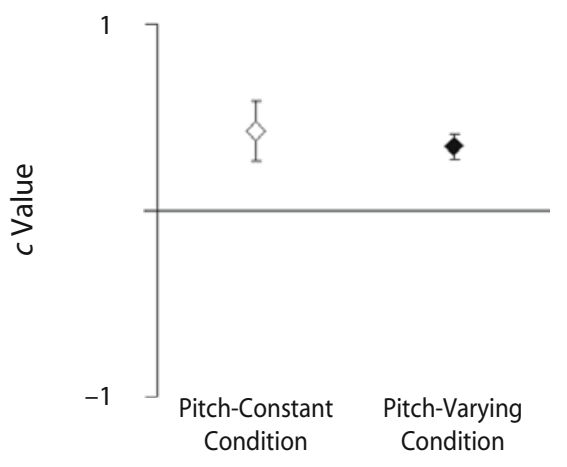

B

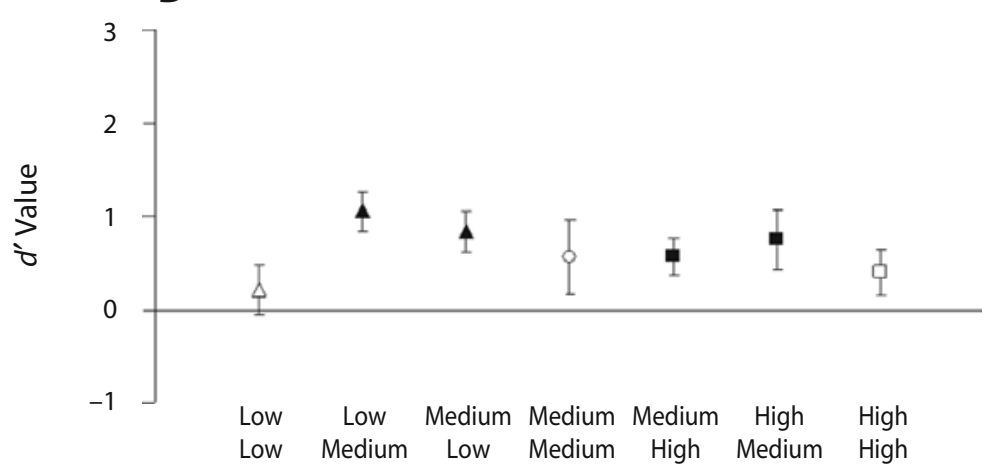

D

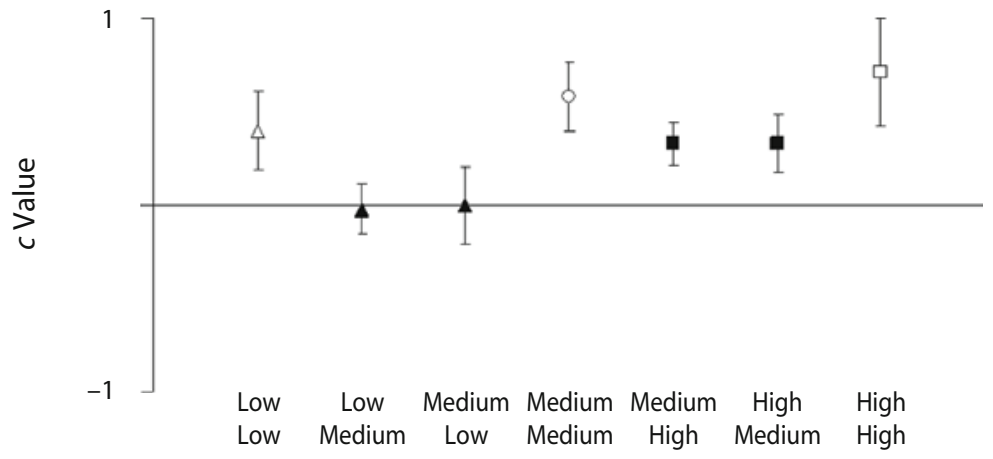

Figure 4. Effect of large pitch variations (4 semitones) on the performance of 9 amusic participants in the duration classification task (Experiment 3). (A) Mean and standard error of the $d^{\prime}$ value in the pitch-constant (PC) and in the pitch-varying (PV) condition. (B) Mean and standard error of the $d^{\prime}$ value for the seven types of intervals presented in the PV condition. (C) Mean and standard error of the $c$ value in the PC and PV conditions. (D) Mean and standard error of the $c$ value for the seven types of intervals presented in the PV condition.

of the perceived duration for intervals bounded by different pitches. However, amusics did not show the association between pitch level and duration as was observed in normal participants in both Experiments 1 and 2. For amusics, time intervals bounded by high- and low-pitched tones were not associated with short and long durations, respectively. This suggests that congenital amusia is associated with abnormal pitch-time association.

Finally, the amusics showed in Experiment 3 a general propensity to respond "short" more often than "long" in both PC and PV conditions, which was not observed in Experiment 2. A possible explanation is that the memory of the standard drifted toward a longer interval. This explanation is in line with adaptive-oscillator models of interval timing (Large \& Jones, 1999; McAuley, 1995) and with the fact that, if IOIs are shorter than $500 \mathrm{msec}$, tempo sensitivity is better for tempo increases than for tempo decreases (McAuley, 1995). Oscillators would adapt their period to the standard IOI of $200 \mathrm{msec}$ during the encoding phase to constitute a reference for the comparison. During the comparison phase, these oscillators would be attracted toward their resting period, which would be longer than the standard IOI of $200 \mathrm{msec}$, leading to an underestimation of test intervals. However, this general propensity to respond "short" was not present with the same participants in Experiment 2, suggesting that this drift is not systematic. This drift might have been due to fatigue in Experiment 3 (which was always performed after Experiment 2).

\section{GENERAL DISCUSSION}

The results of the present study show that congenital amusia is related to an inability to detect fine-grained pitch differences, notably during a duration classification task that does not require attention to pitch variations. Estimates of interval duration are impaired by the presence of quarter-semitone pitch variations in normal participants but not in amusics. It is only when pitch variations are raised to 4 semitones that amusic participants exhibited an interference effect on duration judgments, hence discarding task factors or memory as responsible for their lack of responsiveness to fine-grained pitch differences.

Previous studies have shown that congenital amusics are impaired in pitch processing (Foxton et al., 2004; Hyde \& Peretz, 2004). However, the experimental protocols used in these studies investigated the ability of participants to 
consciously detect fine-grained pitch differences. Therefore, the failure of amusics in these prior studies could have been due to a lack of confidence rather than to a lack of perception. That is why we designed a duration discrimination task that does not require attention to pitch variations in order to assess the real nature of the disorder associated with congenital amusia. Our findings support the notion that congenital amusia is caused by a poor pitch resolution system that affects both covert and noncovert pitch perception.

Furthermore, the present study highlights different aspects of pitch-time interactions. The first aspect is the fact that intervals bounded by different pitches are perceived as being longer than intervals bounded by identical pitches. This effect was observed only in Experiments 1 and 3 and was rather small. The second aspect is a pitchlevel effect. Time intervals bounded by low-pitched tones are more likely to be judged as "long," whereas time intervals bounded by high-pitched tones are more likely to be judged as "short," suggesting a pitch-duration association. This effect was large but was observed only in control participants (Experiments 1 and 2). However, no single explanation at this point can explain these pitchtime effects. Furthermore, these effects might be limited to the context of the experiment. In particular, the fact that pitch variations occurred randomly in the $\mathrm{PV}$ condition could have favored these effects. Jones and Boltz (1989) proposed that, when events are unpredictable, temporal judgments are based on an analytic attending mode, in which attention is placed on details about these events. This would explain that, in our study, duration judgments were influenced strongly by the characteristics of the tones. Further experiments with normal listeners should aim at testing the influence of random presentation on the observed pitch-time effects.

Interestingly, the pitch-level effect on duration judgments was not observed in amusic participants even in the presence of large pitch differences, suggesting that their representation of pitch does not connect with their representation of time. Foxton, Nandy, and Griffiths (2006) have already reported abnormal pitch-time interaction in congenital amusia. They noticed that amusics had difficulties in rhythm discrimination only for the sequences that varied in pitch, suggesting an abnormal pitch-time dependency. Together, this finding and ours suggest that the difficulty of amusics in processing music could arise from a deficit in fine-grained pitch discrimination, which, secondarily, would impair their ability to connect the pitch characteristic of sounds to temporal information. A question for the future is to understand how the brain manages to link pitch with time, and, thereby, to convert sound sequences in music.

\section{AUTHOR NOTE}

We thank our participants for their cooperation. We thank our reviewers, Stephen Handel, Mari Riess Jones, and J. Devin McAuley, for their helpful and constructive comments on the manuscript. This work was supported by grants from the Natural Sciences and Engineering Research Council of Canada, from the Canadian Institutes of Health Research, from a Canada Research Chair, and from the Human Frontier Science Program to I.P. Address correspondence to M. Pfeuty, Laboratoire
d'Imagerie Moléculaire et Fonctionnelle, CNRS (UMR 5231), Université Victor Segalen Bordeaux 2, 146, rue Léo Saignat, Case 117, 33076 Bordeaux Cedex, France (e-mail: micha.pfeuty@u-bordeaux2.fr).

\section{REFERENCES}

Boltz, M. G. (1998). Tempo discrimination of musical patterns: Effects due to pitch and rhythmic structure. Perception \& Psychophysics, 60, 1357-1373.

Brigner, W. L. (1988). Perceived duration as a function of pitch. Perceptual \& Motor Skills, 67, 301-302.

CoHEN, J. (1988). Statistical power analysis for the behavioral sciences (2nd ed.). Hillsdale, NJ: Erlbaum.

Cohen, J., Hansel, C. E. M., \& Sylvester, J. D. (1953). A new phenomenon in time judgment. Nature, $\mathbf{1 7 2}, 901$

Cohen, J., Hansel, C. E. M., \& Sylvester, J. D. (1954). Interdependence of temporal and auditory judgments. Nature, 174, 642-644. doi: $10.1038 / 174642 \mathrm{a} 0$

Crowder, R. G., \& Neath, I. (1995). The influence of pitch on time perception in short melodies. Music Perception, 12, 379-386.

Foxton, J. M., Dean, J. L., Gee, R., Peretz, I., \& Griffiths, T. D. (2004). Characterization of deficits in pitch perception underlying "tone deafness." Brain, 127, 801-810.

Foxton, J. M., Nandy, R. K., \& GrifFiths, T. D. (2006). Rhythm deficits in "tone deafness." Brain \& Cognition, 62, 24-29.

Green, D. M., \& Swets, J. A. (1966). Signal detection theory and psychophysics. New York: Wiley.

Grondin, S., Ivry, R. B., Franz, E., Perreault, L., \& Metthé, L. (1996). Markers' influence on the duration discrimination of intermodal intervals. Perception \& Psychophysics, 58, 424-433.

Grondin, S., \& Rousseau, R. (1991). Judging the relative duration of multimodal short empty time intervals. Perception \& Psychophysics, 49, 245-256.

HenRY, M. J., \& McAuley, J. D. (2009). Evaluation of an imputed pitch velocity model of the auditory kappa effect. Journal of Experimental Psychology: Human Perception \& Performance, 35, 551-564.

Hyde, K. L., Lerch, J. P., Zatorre, R. J., Griffiths, T. D., Evans, A. C., \& Peretz, I. (2007). Cortical thickness in congenital amusia: When less is better than more. Journal of Neuroscience, 27, 1302813032.

Hyde, K. L., \& Peretz, I. (2004). Brains that are out of tune but in time. Psychological Science, 15, 356-360.

Hyde, K. L., Zatorre, R. J., Griffiths, T. D., Lerch, J. P., \& Peretz, I. (2006). Morphometry of the amusic brain: A two-site study. Brain, 129, 2562-2570. doi:10.1093/brain/awl204

IVRY, R. B., \& SchleRf, J. E. (2008). Dedicated and intrinsic models of time perception. Trends in Cognitive Sciences, 12, 273-280.

Jones, M. R., \& Boltz, M. G. (1989). Dynamic attending and responses to time. Psychological Review, 96, 459-491.

Kalmus, H., \& FrY, D. B. (1980). On tune deafness (dysmelodia): Frequency, development, genetics and musical background. Annals of Human Genetics, 43, 369-382.

Large, E. W., \& Jones, M. R. (1999). The dynamics of attending: How people track time-varying events. Psychological Review, 106, 119159.

Lebrun-Guillaud, G., \& Tillmann, B. (2007). Influence of a tone's tonal function on temporal change detection. Perception \& Psychophysics, 69, 1450-1459.

LidjI, P., Kolinsky, R., Lochy, A., \& Morais, J. (2007). Spatial associations for musical stimuli: A piano in the head? Journal of Experimental Psychology: Human Perception \& Performance, 33, 1189-1207.

Loui, P., Guenther, F. H., Mathys, C., \& Schlaug, G. (2008). Action-perception mismatch in tone-deafness. Current Biology, 18, R331-R332.

Macmillan, N. A., \& Creelman, C. D. (2005). Detection theory: A user's guide (2nd ed.). Mahwah, NJ: Erlbaum.

Mandell, J., Schulze, K., \& Schlaug, G. (2007). Congenital amusia: An auditory-motor feedback disorder? Restorative Neurology \& Neuroscience, 25, 323-334.

McAuley, J. D. (1995). Perception of time as phase: Toward an adaptive-oscillator model of rhythmic pattern processing. Unpublished doctoral dissertation, Indiana University. 
OHALA, J. J. (1983). Cross-language use of pitch: An ethological view. Phonetica, 40, 1-18.

Ohala, J. J. (1984). An ethological perspective on common crosslanguage utilization of $F 0$ of voice. Phonetica, 41, 1-16.

Oliveri, M., Vicario, C. M., Salerno, S., Koch, G., Turriziani, P., Mangano, R., ET AL. (2008). Perceiving numbers alters time perception. Neuroscience Letters, 438, 308-311.

Ono, F., \& Kitazawa, S. (2009). The effect of marker size on the perception of an empty interval. Psychonomic Bulletin \& Review, 16, 182-189.

Penner, M. J. (1976). The effect of marker variability on the discrimination of temporal intervals. Perception \& Psychophysics, 19, 466-469.

Peretz, I. (2001). Brain specialization for music: New evidence from congenital amusia. In R. J. Zatorre \& I. Peretz (Eds.), The biological foundations of music (Annals of the New York Academy of Sciences, Vol. 930, pp. 153-165). New York: New York Academy of Sciences.

Peretz, I. (2006). The nature of music from a biological perspective. Cognition, 100, 1-32.

Peretz, I., Brattico, E., Järvenpä̈̈, M., \& Tervaniemi, M. (2009). The amusic brain: In tune, out of key, and unaware. Brain, 132, 12771286

Peretz, I., Champod, A. S., \& Hyde, K. (2003). Varieties of musical disorders: The Montreal Battery of Evaluation of Amusia. In B. Avanzini, C. Faienza, \& D. Minciacchi (Eds.), The neurosciences and music (Annals of the New York Academy of Sciences, Vol. 999, pp. 58-75. New York: New York Academy of Sciences.

Pratt, C. C. (1930). The spatial character of high and low tones. Journal of Experimental Psychology, 13, 278-285.

Rusconi, E., Kwan, B., Giordano, B. L., Umiltà, C., \& ButterWORTH, B. (2006). Spatial representation of pitch height: The SMARC effect. Cognition, 99, 113-129.

SHIGENO, S. (1993). The interdependence of pitch and temporal judgments by absolute pitch possessors. Perception \& Psychophysics, 54, 682-692

Stevens, S. S. (1934). The attributes of tones. Proceedings of the National Academy of Sciences, 20, 457-459.

Wearden, J. H., Wearden, A. J., \& Rabbitt, P. M. A. (1997). Age and IQ effects on stimulus and response timing. Journal of Experimental Psychology: Human Perception \& Performance, 23, 962-979.

Xuan, B., Zhang, D., He, S., \& Chen, X. (2007). Larger stimuli are judged to last longer. Journal of Vision, 7, 1-5.

(Manuscript received January 29, 2009; revision accepted for publication October 16, 2009.) 